the improvement of selective diagnostic nutrient media for the detection of $T$. vaginalis is so important.

The purpose of the study was to increase the selectivity and growth properties of the nutrient medium for the $T$. vaginalis detection. It was necessary to select a concentration of amphotericin B in media with different content of horse serum and peptone enzymatic, which inhibits the growth of Candida spp., but doesn't affect the growth of $T$. vaginalis.

For the research, an experimental mediums (based on the SVT medium (RU FSS No. 2009/05982), produced by the Pasteur Institute) were prepared with different content of horse serum $(10 \%, 20 \%, 40 \%)$, half of which were with enzyme peptone $(12.8 \mathrm{~g} / \mathrm{l})$ and all of them without antimycotics. Two dilutions of amphotericin $\mathrm{B}$ in the range of concentrations of $0.5-50 \mu \mathrm{g} /$ $\mathrm{ml}$, as well as fluconazole at a concentration of $264 \mathrm{mg} /$ $\mathrm{ml}$ (as in the SVT) were introduced into all experimental media. The strains of $T$. vaginalis (T1, T5, T7, T11) from the Pasteur Institute collection in concentration $0.5 \times 10^{6}$ cells/ml and the standard strain Candida albicans ATCC 24433 in concentration $10^{7}$ cells $/ \mathrm{ml}$ were sown in all medias in three recurrence. The incubation temperature was $35 \pm 1^{\circ} \mathrm{C}$. Counting the number of cells was carried out using the Goryaev chamber every day for a week. After 24 hours, C. albicans were sowed onto the Müller-Hinton agar for testing the suppression of their viability with subsequent microscopy.

It was revealed, that optimal accumulation of $T$. vaginalis $\left(2.5 \times 10^{6}\right.$ cells $\left./ \mathrm{ml}\right)$ and inhibition of $C$. albicans occurs at concentration of amphotericin B (2-0.5) $\mu \mathrm{g} / \mathrm{ml}$. The activity of fluconazole to $C$. albicans in these media was low. It should be noted, that in medias with a high content of horse serum, the accumulation of T. vaginalis increased sharply on the second day of the study, and their resistance to high concentrations of antimycotic (up to $20 \mu \mathrm{g} / \mathrm{ml}$ ) was also observed. However, the viability of the cells was reduced in contrast to media with $10 \%$ horse serum and low concentrations of amphotericin B. The addition of enzymatic peptone to experimental media did not reveal a significant difference in the growth properties.

According to the results, an experimental medium containing $10 \%$ horse serum with amphotericin B in concentration $2 \mu \mathrm{g} / \mathrm{ml}$ was chosen for detection and accumulate $T$. vaginalis doi: 10.15789/2220-7619-2018-4-9.2

\section{ANALYSIS OF THE PHAGE SENSITIVITY OF MICROORGANISMS OF A MICROBIOTA OF A VAGINA}

I.S. Polishchuk, A.V. Aleshukina, I.S. Aleshukina, T.I. Tverdokhlebova

Rostov Research Institute of Microbiology and Parasitology, Rostov-on-Don, Russia

Recently in connection with growth of detection of antibiotic resistant cultures, for treatment of infectious diseases even more often recommend to use bacteriophages. Bacteriophages don't give side effects in comparison with antibiotics and work is specific on microorganisms and exist in different pharmaceutical forms: liquid, gel and tableted. The solution of a question of application of a phage has to be based on results of testing of activity of medicine.

The aim of the study was comparative analysis of two options of phagus medicines for definition of a phagus sensitive of microorganisms of a microbiota of a vagina.
50 women who have addressed to laboratory on an outpatient basis for the purpose of receiving a bacteriological research of a vaginal microbiota have been examined. Bacteriological researches were conducted according to the standard recommendations. For identification of species of bacteria by MALDI-TOF MS method used a desktop mass spectrometer of Microflex with the MALDI Biotyper library (Bruker Daltonics Germany). As the tested medicines applied polyvalent liquid and gel forms of bacteriophages. The modified technique where the bacteriophage was applied with a print on culture a bacteriological loop $(\mathrm{cm} \mathrm{d}=0.5)$ bent at an angle of $90^{\circ}$ lehas been developed for a gel form of a bacteriophage. Assessment of lytic activity of a phage was carried out on a five-point scale (by quantity of "crosses").

The sensitivity to bacteriophages has been defined at 45 women with violation of a microbiota of $\mathrm{V}$. At the same time the high sensitivity to a liquid form of a bacteriophage has been found in 6 patients (13\%). To a gel form the high sensitivity has been defined at 39 patients $(87 \%)$, coincidence cases on sensitivity at both bacteriophages weren't observed.

The parallel research of sensitivity of microflora to liquid and gel forms at a bacterial vaginosis has shown that in $87 \%$ microorganisms were sensitive to a gel form while the sensitivity to liquid bacteriophages has been found in $13 \%$ of the bacteria inhabiting the offered modification of a research of activity of bacteriophages on a gel basis allows to dose bacteriophages in this pharmaceutical form and to receive comparable results with a classical technique.

\section{S. AUREUS/C. ALBICANS MONO- AND DUAL-SPECIES BIOFILMS}

\section{O.N. Postnikova, D.V. Chepurina}

V.I. Vernadsky Crimean Federal University, Simferopol, Russia

Several studies have reported the co-isolation of $S$. aureus and $C$. albicans from numerous biofilm-associated diseases. These data indicate that these organisms have the capacity to interact with one another at the molecular level. The possibility of the development of polymicrobial biofilms, consisting of both fungi and bacteria, should be considered in pathogenesis of various infections.

The aim of our work was study of mono-and dual-species $S$. aureus $/ C$. albicans biofilms, evaluation of distinctions between clinical and standard streins biofilms of both in static assays.

In the work used standard strains of $S$. aureus 25923 ATCC and C. albicans CCM 885, as well as clinical strains of them, isolated from patients with acute otitis media (S. aureus U14) and also from healthy carrier (S. aureus 609, C. albicans 609).

Overnight cultures was diluted 1:100 into fresh medium for biofilm assays. Static biofilm assays of O`Toole G. and Kolter R. (1998) in a 96 well dish was used. Biofilms were formed by adding both organisms 1:1 to either microtiter plates. The plate was then grown statically at $37^{\circ} \mathrm{C}$ overnight. The cultures removed with a multichannel pipette plate, the plate was rinsed 3-4 times with water, a $0.1 \%$ solution of crystal violet in water was added of to each well, incubated for 15 minutes at RT. Then $200 \mu \mathrm{L}$ of $95 \%$ ethanol was added to each well and the plate was left to stand on the bench for 30 minutes. Finally the plate was read with a microplate reader Multiskan at $620 \mathrm{~nm}$.

Biofilms density of Staphylococcus cultures was the highest in the clinical isolate $S$. aureus U14 (24\% more 
than standard strains $S$. aureus 25923 ATCC). Density of C. albicans 609 biofilm exceed the density of the standard strain C. albicans CCM 885 biofilm at $14.5 \%$. However, dual bacteria-fungal biofilm $S$. aureus U14 + C. albicans 609 exceed density of biofilm $S$. aureus 25923 ATCC $+C$. albicans CCM 885 more than twice (208\%). Dual-species biofilm isolated from throat healthy media, $S$. aureus $609+C$. albicans 609 , did not differ in density from dual-species biofilm of reference cultures.

Thus, when coupled with cultivation yeast $C$. albicans in vitro aggressiveness of $S$. aureus clinical strain was increased. Carrier state yeast fungi, therefore, could serve as a risk factor for developing a more dense staphylococcal biofilm on the mucous membranes and worsen the course of the disease.

\section{MICROBIOLOGICAL MONITORING} OF THE RESISTANCE OF HOSPITAL BACTERIAL FLORA WITHIN THE SYSTEM OF PREVENTION OF HEALTHCARE-ASSOCIATED INFECTIONS

N.V. Shestopalov ${ }^{1,2}$, T.V. Gololobova ${ }^{1,2}$, L.S. Fedorova ${ }^{1}$, A.A. Serov ${ }^{1}$, A.I. Ruleva ${ }^{3}$

${ }^{1}$ Research Institute of Disinfectology, Moscow, Russia $;{ }^{2}$ FSBEI FPE RMACPE MOH Russia, Moscow, Russia ; ${ }^{3}$ Treatment and Rehabilitation Center, Moscow, Russia

Effective implementation of disinfection activities in healthcare organizations (HCO) plays a great and, sometimes, even a critical role in the prevention of healthcare-associated infections (HAI). Formation and spread of microorganisms which are resistant to used chemical disinfectants in HCO substantially reduce the effect of disinfection measures. This, in turn, is the common reason of the high level of HAI incidence. The problem is aggravated by the development of antibiotic resistance among the strains which are resistant to DA (cross-resistance)

According to the requirements of Sanitary Regulations and Norms 2.1.3.2630-10, it is necessary to conduct monitoring of hospital bacterial flora sensitivity to disinfection agents (DA).

The assessment of sensitivity of microorganisms isolated from the objects of intrahospital environment of intensive care, intensive therapy and surgical units -20 strains (29\%), and from the pathological loci of in-patients 50 strains $(71 \%)$ resistant to various groups of antibiotics (K. pneumonia, A. baumannii, P. aeruginosa, P. mirabilis, S. maltophilia) - was carried out.

Testing was conducted according to the method described in Methodical Guidelines 3.5.1.3438-17 "Assessment of sensitivity to disinfection agents demonstrated by microorganisms circulating in healthcare organizations". To compare the resistance of hospital microorganisms with that of the microorganisms from the collection which demonstrate standard resistance to DA a collection strain $P$. aeruginosa ATCC 27853 was used.

It was determined that 11 strains were resistant to cationic surface-active agents, 10 strains - to active oxygen, 6 strains - to the combination of quaternary ammonium compounds and active oxygen. $27(38.7 \%)$ out of 70 strains of microorganisms isolated from external environment objects and patients were resistant to used DA.

The identity of resistant strains isolated from external environment and patients serves as evidence of cross-contamination and leads to the spread of resistant strains among patients which, in turn, determines the need to improve the organization of preventive activities. doi: $10.15789 / 2220-7619-2018-4-9.24$

GENETIC VARIANTS OF RESISTANCE

\section{DETERMINANT TO SILVER IN EPIDEMIC STRAINS} OF ACINETOBACTER BAUMANNII

\section{A.P. Solomennyi ${ }^{1}$, N.A. Zubareva}

${ }^{1}$ Institute of Ecology and Genetics of Microorganisms, Federal Research Centre of Ural Branch of RAS, Perm, Russia,

${ }^{2}$ E.A. Wagner State Medical University, Perm, Russia

Silver-containing dressings are widely used in burns and leg ulcers for prevention and treatment infection. Heavy metal pump CzcA of RND transporters family predicted roles in the efflux both toxic cations including silver and some antibiotics from bacterial cells. Molecular epidemiology and surveillance of outbreaks for last decades indicate that the most resistant Acinetobacter baumannii belong to two globally disseminated clonal lineages, GC1 and GC2. Strains of GC2 are epidemic as for Far East and Indochina. Our study was designed in order to clarify an evolution of silver resistance determinant in clinical A. baumannii.

Multiple-resistant strains from different geographic locations were selected. List of strains included $A$. baumannii AYE (GC1, epidemic in France for past years) and strains of GC2: ACICU isolated in an outbreak (Italy), sturdy-biofilm forming 1656-2 (South Korea), LY9 and BJ5 recovered in Southern and Northern China hospitals, consequently and strain of sequence-type ST2 (Institut Pasteur typing scheme) endemic in Perm in 2010-2011. Sequences of $c z c A$ were retrieved from GenBank database for in silico comparative analysis using BLAST.

Surprisingly, $c z c A$ gene on chromosomes of $A$. baumannii $\mathrm{GC} 1$ and $\mathrm{GC} 2$ spread over the world is presented as two prevailing alleles only (allele 1 in AYE, ACICU, 1656-2 and allele 2 in LY9, BJ5 and Perm). Even di- and multinucleotide variants on positions $213-215$ ACC or TTT, 897-898 TG or AC, 945-948 CCGT or TAAA and 951-952 AG or TA have been related to distinct allele (nucleotide numbers from start codon ATG).

The important mechanism of $A$. baumannii survival under silver presence is expressed in an extreme decrease in the genetic heterogeneity of encoding sequence.

This study was supported by the Federal research programme under state registration number 01201353247.

\section{COMMENSAL STRAINS OF ESCHERICHIA COLI AND} BETA-LACTAM RESISTANCE

L.V. Suzhaeva, S.A. Egorova, L.A. Kaftyreva

St. Petersburg Pasteur Institute, St. Petersburg, Russia

According to the World Health Organization, the antimicrobial resistance (AMR) remains a huge worldwide problem of our time which we yet have to overcome. One of the ways to contain the antibiotic resistance is to monitor a circulation of resistant strains of microorganisms, as well as genes that determine the AMR. Studies in recent years have shown a high level of resistance in Klebsiella pneumoniae and Escherichia coli, the causative agents of nosocomial and community-acquired infections. However, resistant strains also may be a part of gastrointestinal microbiota in healthy individuals.

Antimicrobial susceptibility of 511 commensal E. coli strains isolated from faeces of children in age groups from 1 month to 17 years old living in St. Petersburg to 9 groups of antibiotics have been studied by disco-diffusion method. The resistance mechanisms among strains insusceptible 